\title{
Der «Processus universalis» nach Michael Sendivogius
}

Zur Entstehungsgeschichte einer neuzeitlichen Variante

des alchimischen Grossen Werkes

Rolf Gelius

\section{Summary}

The "Processus D. Michaelis Sendivogii super centrum universi, seu Sal centrale", printed by Johann Joachim Becher in his "Chymischer Glücks-Hafen" (1682), originated from a cryptic-alchemical recipe in Sendivogius' treatise "De Lapide Philosophorum" (1604). The further development of the initial procedure passed through an intermediate stage and culminated in an alchemical text which was composed before 1632, probably at the court of Moritz of Hessen-Kassel. On favourable reaction conditions this novel "Processus universalis" might yield coloured $\mathrm{Ag} / \mathrm{Au} / \mathrm{Cl}$ coordination compounds of the $\mathrm{Ag}\left[\mathrm{AuCl}_{4}\right]$ type.

\section{Zusammenfassung}

Der «Processus D. Michaelis Sendivogii super centrum universi, seu Sal centrale», abgedruckt von Johann Joachim Becher in seinem «Chymischer Glücks-Hafen» (1682), geht auf eine spekulativ-alchimische Rezeptur in Sendivogius' Traktat «De Lapide Philosophorum» (1604) zurück. Die weitere Entwicklung der Originalprozedur führte über Zwischenstufen zu einem vor 1632 entstandenen alchimischen Manuskript, das wahrscheinlich am Hof des Landgrafen Moritz von Hessen-Kassel verfasst wurde. Dieser weiterentwickelte «Processus universalis» sollte unter günstigen Reaktionsbedingungen zur Bildung farbiger $\mathrm{Ag} / \mathrm{Au} / \mathrm{Cl}$-Komplexverbindungen des Typs $\mathrm{Ag}\left[\mathrm{AuCl}_{4}\right]$ führen.

Doz. Dr. Rolf Gelius, Loissiner Wende 1 B, D-17491 Greifswald, Deutschland 
Johann Joachim Becher hat 1682 in seiner «Grossen Chymischen Concordantz» eine «Collection von funffzehen hundert Chymischen Processen... auß den besten Manuscriptis und Laboratoriis ...» abgedruckt ${ }^{1}$. Darunter befindet sich auch ein «Processus D. Michaelis Sendivogii super centrum universi, seu Sal centrale», ein neuartiges und interessantes Verfahren zur Gewinnung des Lapis Philosophorum. Er wird in zwei deutsch-lateinischen Druckfassungen (Drfg. Becher I und II $)^{2}$ vorgestellt, von denen die erste Variante 10 Kapitel sowie eine theoretische Einleitung umfasst, die zweite 9 kürzere Abschnitte. Beide Varianten stimmen inhaltlich weitgehend überein.

\section{Die Chemie des «Processus universalis» nach Sendivogius}

Nach van Nieuwenburg ${ }^{3}$ könnten die Alchimisten bei ihren tausendfach variierten Versuchen zur Gewinnung des Lapis Philosophorum auf einen wirklichen «Goldsamen» gestossen sein, nämlich auf das rote Silbertetrachloroaurat (III), $\mathrm{Ag}\left[\mathrm{AuCl}_{4}\right]^{4}$. Diese Substanz enthält 44,1\% Gold und 24,2\% Silber. Mit ihr wäre allerdings nur ein «Particular» möglich, die einmalige «Transmutation» (Bildung einer goldfarbenen Legierung aus unedlen Metallen), kein echter «Processus universalis». Leider gibt der Autor keine Beispiele für solche Präparationen aus dem alchimischen Schrifttum.

Der «Processus universalis» nach Sendivogius, vorgestellt in den Becherschen Druckfassungen, sollte unter günstigen Umständen tatsächlich zur Bildung kristalliner Silber-Gold-Chlorkomplexe führen. Ihre Bildung würde hier jedoch vorwiegend nasschemisch erfolgen, während van Nieuwenburg ihre Trocken-Synthese (bei $>350{ }^{\circ} \mathrm{C}$ in Gegenwart von Sublimat, $\mathrm{HgCl}_{2}$ ) diskutiert.

1 J. J. Becher: Chymischer Glücks-Hafen, Oder Grosse Chymische Concordantz und Collection / Von funffzehen hundert Chymischen Processen: Durch viel Mühe und Kosten auß den besten Manuscriptis und Laboratoriis in diese Ordnung / ... zusammen getragen. Franckfurt: J. G. Schiele 1682, S. 231-240.

2 Drfg. Becher I umfasst die Seiten 231-236, Drfg. Becher II die Seiten 236-240.

3 C.J. van Nieuwenburg, in: South African Ind. Chemist 17 (1963), S. 132-133.

$4 \mathrm{Ag}\left[\mathrm{AuCl}_{4}\right]$ entsteht aus AgCI und überschüssiger $\mathrm{H}\left[\mathrm{AuCI}_{4}\right]$-Lösung in konzentrierter Salpetersäure beim Kochen auf dem Wasserbad oder durch direkte Fällung von $\mathrm{H}\left[\mathrm{AuCI}_{4}\right] \mathrm{mit}$ salpetersaurer $\mathrm{AgNO}_{3}$-Lösung. Es ist ein mikrokristallines, schweres, orangerotes Pulver von diamantartigem Glanz, an trockener Luft stabil und lichtbeständig. Hydrothermale Rekristallisation aus Salpetersäure $\left(120^{\circ} \mathrm{C}\right)$ liefert rote, monoklinprismatische Einkristalle. Bei 230-270 ${ }^{\circ} \mathrm{C}$ erfolgt Zersetzung zu einer dunkelbraunen, hygroskopischen Schmelze. Vgl. $F$. Hermann, in: Ber. dtsch. chem. Ges. 27 (1894), S. 596/97; N. Parravano u. G. Malquori, in: Gazz. chim. ital.56 (1926), S. 13-19, hier S. 14; W. Werner u. J. Straehle, in: Z. Naturforsch. 32 B (1977) 7, S. 741-744, hier S. 741; C. R. Hall u. M. J. A. Smith, in. J. Phys. Chem. Solids 42 (1981), S. 421-427, hier S. 423. 
Bei der Beschreibung des Arbeitsablaufes folgen wir Drfg. Becher I:

1. Extraktion von «Sal nitri Philosophorum» (Kalksalpeter, $\mathrm{Ca}\left(\mathrm{NO}_{3}\right)_{2} \cdot 4 \mathrm{H}_{2} \mathrm{O}$ ) aus «terra virginea» ${ }^{5}$ nach Arbeitsweise der gewerblichen Salpetersieder ${ }^{6}$ und thermische Zersetzung des Salzes zu nitrosen Gasen, die zu konzentrierter Salpetersäure verarbeitet werden. Trockene Destillation der «terra virginea» liefert ein weisses Sublimat (Ammonsalze), Eindicken des wässerigen Extrakts des Glührückstands ein «Sal fixum» (Kochsalz).

2. Vereinigung dieser drei Komponenten (trium salium conjunctio) zu Königswasser (mit $\mathrm{NaCl}$ und $\mathrm{NH}_{4} \mathrm{Cl}$ als Haupt-Chlorlieferanten), dem «warhafften Menstruum universale». Auflösung von 1 Teil Naturgold ${ }^{7}$ in 10 Teilen des Menstruum, wobei das Gold als $\mathrm{H}\left[\mathrm{AuCl}_{4}\right]$ in $\mathrm{Lö}^{-}$ sung geht und sich eine Erde absetzt $\left(\mathrm{AgCl}\right.$ oder ein $\mathrm{AuCl}_{3} / \mathrm{Ag}\left[\mathrm{AuCl}_{4}\right] / \mathrm{NH}_{4} \mathrm{Cl}$-Tripelsalz ${ }^{8}$ vom Silbergehalt des Goldes).

3. Die dekantierte Lösung wird in einem hermetisch verschlossenen Glaskölbchen in einen Athanor eingesetzt und dort jeweils mehrere Wochen bei steigenden Feuergraden bis zur Rotfärbung erhitzt. In der Mitte des Glases zeigt sich dann ein «granum ad instar rubini rubicans magnitudine lentis ... hoc granum est semen 〈Sol〉is»?

In einer zusätzlichen Operation (compositio magni operis), deren Sinn schwer verständlich ist, wird dieser «Goldsamen», jetzt als «lebendiges Gold» bezeichnet, $\mathrm{zu}$ einem durchsichtigen roten Pulver umgeformt, vielleicht durch Rekristallisation. Es folgen die in der Alchimia transmutatoria üblichen Verfahrensstufen des Grossen Werkes: Multiplikation, Fermentation und Projektion des fermentierten Lapis auf Quecksilber.

Neben den genannten Chloroauraten ist bei dieser Präparation auch die Bildung von Hydroxo- und Oxochloroauraten möglich, z.B. des gelben $\mathrm{Ag}_{2}\left[\mathrm{AuOCl}_{3}\right]$, wenn der Säuregehalt der Lösung zu gering ist. Das Reaktionssystem wird weiterhin dadurch kompliziert, dass die Goldsalze bei höherer Temperatur zerfallen, so $\mathrm{AuCl}_{3}$ ab $170^{\circ} \mathrm{C}$ nach $\mathrm{AuCl}_{3} \rightarrow \mathrm{AuCl}+\mathrm{Cl}_{2}$ und $\mathrm{AuCl}$ ab $200{ }^{\circ} \mathrm{C}$ nach $2 \mathrm{AuCl} \rightarrow 2 \mathrm{Au}+\mathrm{Cl}_{2}$. $\mathrm{AuCl}$ ist gelb, ebenso sein Alkalikomplex $\mathrm{Na}\left[\mathrm{AuCl}_{2}\right]$, feinverteiltes Gold schwarz. Somit kann man vom Gesamtprozess annehmen, dass er die Hauptfarben der alchimischen Transmutation

5 Gemeint ist hier offensichtlich natürliche Salpetererde, die stets grössere Mengen Kochsalz sowie etwas Ammonsalz (Ammonchlorid, Ammoncarbonat/-carbamat) enthält.

6 Die beste zeitgenössische Vorschrift stammt von L. Ercker: Beschreibung der Allerfürnemisten Mineralischen Ertzt vnnd Bergkwercks arten /..., Franckfurt am Mayn 1580 (neuhochdeutsche Übertragung von P. R. Beierlein, in: Freiberger Forsch.-H. D34 (1960), hier S. 257-270.

7 Der Prozess kann nur erfolgreich sein, wenn das Naturgold ungereinigt, d. h. silberhaltig ist. Siebenbürgisches Naturgold, ein häufig verwendetes Alchimistengold, enthielt 15 bis $30 \%$ Silber. Vgl. C. Doelter u. H. Leitmeier (Hrsg.): Handbuch der Mineralogie, Bd. III. 2, Dresden/Leipzig 1926. S. 188/89.

8 Das Tripelsalz bildet sich aus $\mathrm{AgCI}$ beim Stehenlassen mit $\mathrm{NH}_{4} \mathrm{CI}$-haltiger salpetersaurer $\mathrm{H}\left[\mathrm{AuCI}_{4}\right]$-Lösung. Nach Reinigung tief dunkelrote, orthorhombische Prismen von hohem Lichtbrechungsvermögen und starkem Pleochroismus. Vgl. W. B. Pollard, in: J. chem. Soc. (London) 117 (1920), S. 99-103, hier S. 101/02; H. L. Wells, in: Amer. J. Sci. [5] 3 (1922), S. $257-259$.

9 Drfg. Becher I, S. 235.- Anmerkung: Alchimische Symbole wurden aufgelöst; deutsche bzw. lateinische Übertragungen stehen in Scherenklammern. 
zeigt: Schwarz (feinverteiltes Gold), Weiss (Ammonsalz-Sublimat), Gelb $\left(\mathrm{AuCl}, \mathrm{Na}\left[\mathrm{AuCl}_{2}\right], \mathrm{Ag}_{2}\left[\mathrm{AuOCl}_{3}\right]\right)$ und schliesslich Rot des «Goldsamens» $\mathrm{Ag}\left[\mathrm{AuCl}_{4}\right]$.

Der hier vorgestellte Reaktionsablauf ist offensichtlich an zwei Voraussetzungen gebunden: (1) Anwesenheit von genügend $\mathrm{AgCl}$ in der Phiole; (2) das Kölbchen darf - zumindest in der Endphase der Reaktion - nicht «hermetice» verschlossen sein, denn Bechers Präparationsvorschrift spricht nicht von der Abscheidung des «Goldsamens» aus wässeriger Phase, sondern von einem $« \ldots$ pulverem rubicundum, qui in circuitu hujus grani est $»^{10}$.

\section{Zur Entstehungsgeschichte des «Processus universalis» nach Sendivogius}

Titelgestaltung und theoretische Grundlagen der Becherschen Druckfassungen haben einige neuere polnische Chemiehistoriker (besonders R. Bugaj $^{11}$ und Z. Szydlo ${ }^{12}$ ) zu der Annahme geführt, der «Processus ... super ... Sal centrale» stamme von Michael Sendivogius (1566-1636) persönlich. Er habe ihn bei seinem Prager Aufenthalt 1598 niedergeschrieben und das Manuskript Kaiser Rudolf II. übergeben. Nach späteren redaktionellen Bearbeitungen in der kaiserlichen Alchimistenszene sei es dann in die Hände Bechers gelangt.

Eine genauere Prüfung der Sendivogius-Schriften erweckt jedoch ernsthafte Bedenken gegen diese Hypothese.

Sendivogius' echte Traktate sind ausschliesslich lateinisch abgefasst und unter Pseudonym (Anagramm) veröffentlicht; nach Diktion und Inhalt gehören sie der spekulativen Alchimie an. An keiner Stelle finden wir in ihnen konkrete chemische Rezepturen (siehe unten!). Soweit aus der fraglichen Zeit (vor 1600) eigenhändige deutschsprachige Briefe von Sendivogius vorliegen, sind sie in unbeholfenem Deutsch geschrieben ${ }^{13}$ - ein Hinweis, dass

10 Drfg. Becher I, S. 235.- Es dürfte sich um Hydrolyse- und Zersetzungsprodukte der SilberGold-Chlorokomplexe handeln, vermischt mit $\mathrm{NaCl}$ und feinverteiltem Gold sowie (falls vorhanden) mit den Kupferverunreinigungen des Naturgoldes.

11 R. Bugaj: (a) Michal Sędziwój (1566-1636). Życie i pisma. Wroclaw/Warszawa/Kraków 1968, S. 182-195; (b) Ders., in: Kwart. Hist. Nauki Tech. (1986) 3/4, S. 749-780, hier S. 756 u. 760/61; (c) Ders., Hermetyzm. Wroclaw/Warszawa/Kraków 1991, S. 243-246.

12 Z. Szydlo, in: Ambix 40 (1993) 3, S. 129-146, hier S. 131-133 u. 140-142.

13 Zwei Briefe von Sendivogius aus dem Jahre 1597 an Kaiser Rudolf II. (Prag, 10. 2. 1597) und an Hans Popp, Obersten Kammerdiener des Kaisers und Burggrafen zu Eger (o. O. [Prag], 10. 2. 1597), beide unterschrieben mit «Michael Sedzimir», dem Geburtsnamen des Alchimisten, sind offensichtlich Kanzleikopien; vielleicht wurde auch der Briefstil etwas geglättet: Oesterr. Haus-, Hof- u. Staatsarchiv Wien, Familienkorrespondenz A, Briefe an Kaiser Rudolf II., Kasten 4, B1. 271-274. 
er diese Sprache damals nicht ausreichend beherrschte ${ }^{14}$. Die Versuchsbeschreibungen aus dem «Processus ... super ... Sal centrale» sind mit dem Briefstil des polnischen Alchimisten nicht vergleichbar.

In den gedruckten lateinischen Schriften von Sendivogius findet sich nur eine Rezeptur, die in Beziehung zu Bechers «Processus universalis» steht. Sie gründet sich auf die Sendivogianische Lehre vom «philosophischen Salpeter» (sal petrae Philosophorum, mercurius aeris), der sich, von den Strahlen der Gestirne generiert, in der Erdoberfläche anreichert, und wurde erstmals $1604 \mathrm{im}$ «Tractatus de Lapide Philosophorum» (spätere Auflagen unter dem Titel «Novum Lumen Chymicum») publiziert ${ }^{15}$. Diese Vorschrift ist in spekulativ-alchimischer Manier abgefasst, mit diffusen, vieldeutigen Stoff- und Operationsbezeichnungen:

Überschüssige «terra nostra» (als Träger des philosophischen Salpeters) wird mit «aurum nostrum vivum «et> non vulgaris» sowie dto. «argentum ...» in «ignem nostrum» gesetzt, das Reaktionsprodukt (liquor siccus) in «mercurium Philosophorum» gelöst, der Löserückstand (humidum radicale metallicum) mit einem aus «terra nostra» gewonnenen «aqua salis nitri» versetzt und das Gemisch einer Wärmebehandlung (igne putrefactionis <et> generationis) unterworfen. Dabei sollen nacheinander die Farben des Pfauenschwanzes, dann Schwarz, Grün und Braun erscheinen; schliesslich erhält man ein «aquam fere rubram».

An dieser Stelle vermerkt der Autor:

«Hucusque peruenit mea experientia, non plus possum, non plus inveni.»

Es ist schwer vorstellbar, dass diese verschwommene Anleitung mehr als Spurenmengen farbiger Metallverbindungen lieferte. Sie mag aber für praktisch arbeitende Alchimisten Anreiz gewesen sein, die Arbeitsvorschrift zu konkretisieren und zu erweitern. Tatsächlich fand ich in deutschen Bibliotheken insgesamt acht überwiegend deutschsprachige Manuskripte, die man als Entwicklungsstufen von Sendivogius' Originalrezeptur auffassen kann.

Die Handschriften sind in Tabelle 1 aufgelistet; sie lassen sich zwanglos in drei Gruppen aufteilen:

1. Manuskripte, die sozusagen die Urfassung des «Processus universalis» nach Sendivogius wiedergeben: K I und H I. Beide führen als Quelle den Arzt und Alchimisten Jacob Alstein an, der in den ersten Jahrzehnten des

14 Auch ein eigenhändiger «Kortzer bericht wie mir michaeli Sendiuogio Von ... in landt zu Wirtemberg widerfahren» aus dem Jahre 1606, Teil einer Supplik an den König von Polen und Schweden, zeigt im Vergleich zu den vorstehend genannten Briefen kaum stilistische Verbesserungen: Hauptstaatsarchiv Stuttgart, Bestand A 47, Bü 8, Nr. 6.

15 «Divi Leschi genus amo» (d. i. Michael Sendivogius): Novum Lumen Chymicum, e naturae fonte et manvali experimentia depromptum, et in duodecim Tractatus diuisum: Cui accessit, Dialogus Mercurij, Alchymistae \& naturae, perquam utilis. Coloniae:Antonius Boetzer 1614, Tractatus undecimus, S. 37/38, u. Conclusio, S. 50/51. 


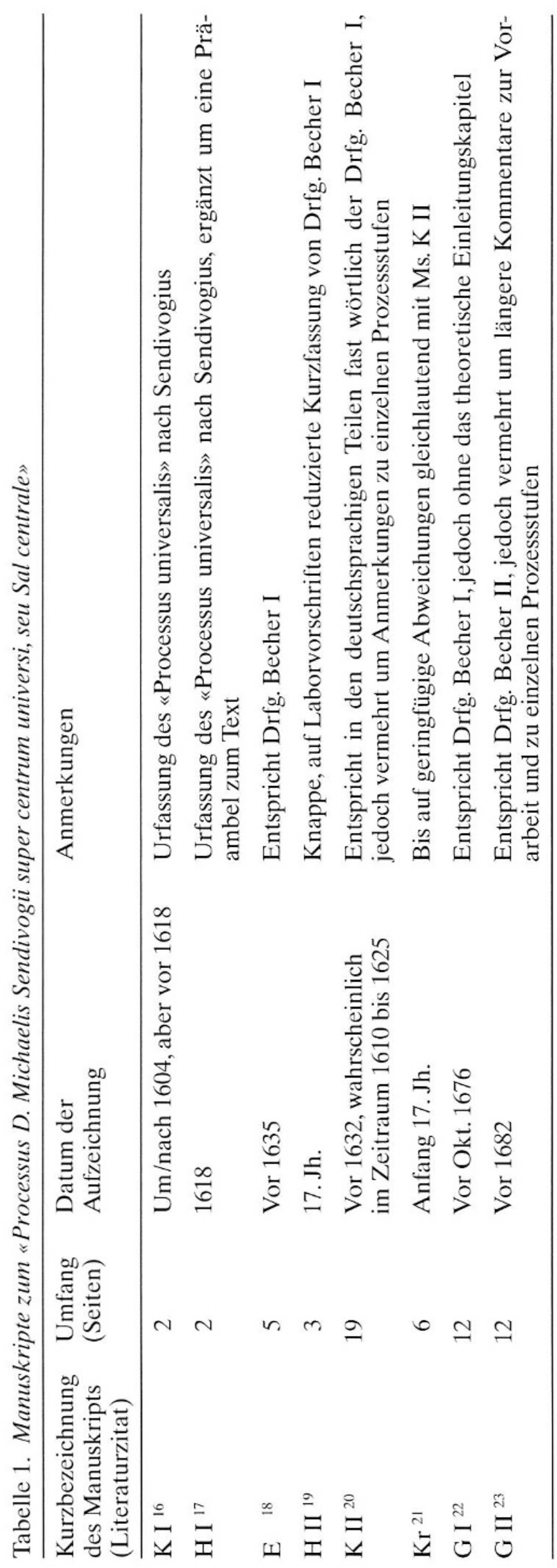

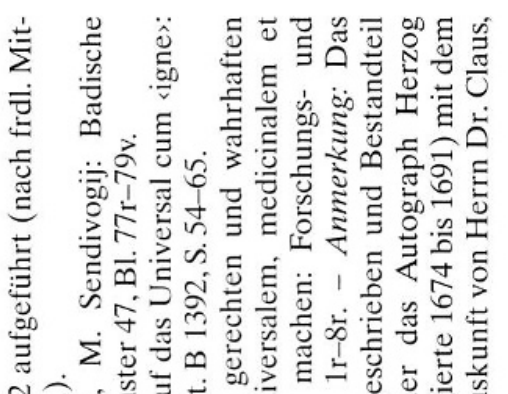

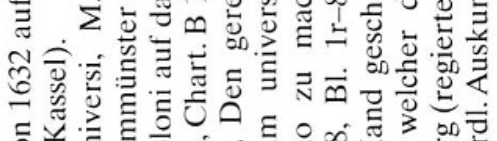

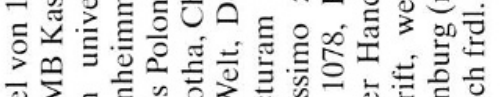

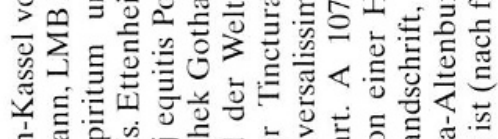

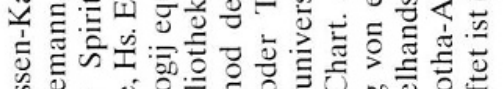

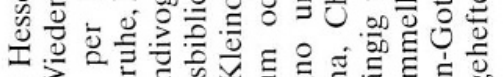

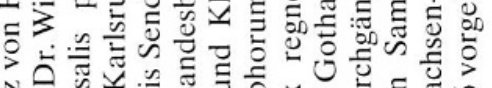

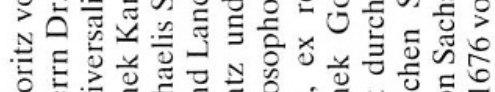

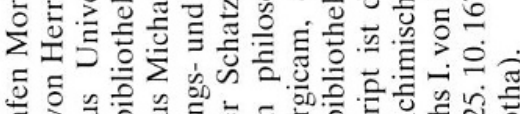

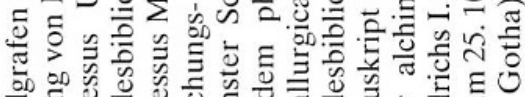

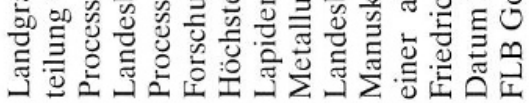
$\overline{4}$ ก

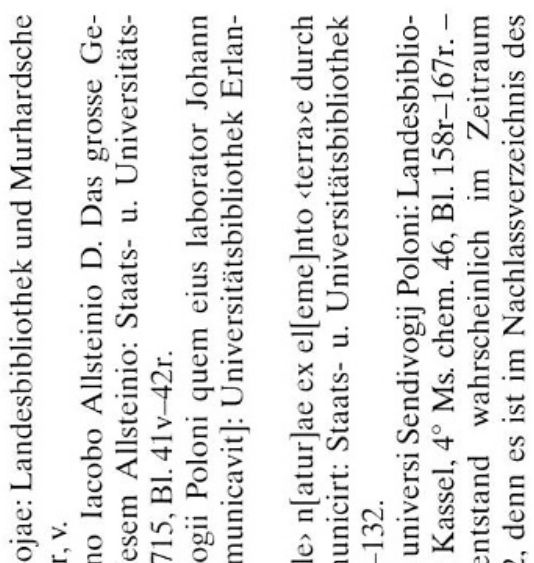

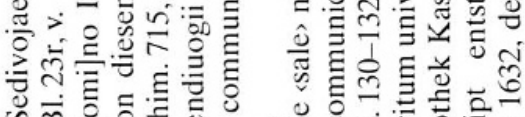

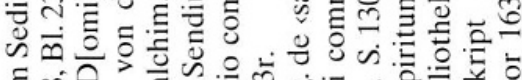

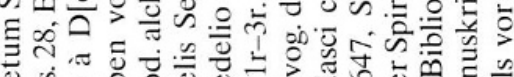

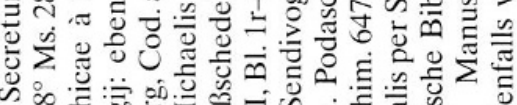

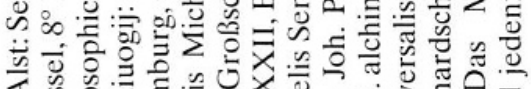

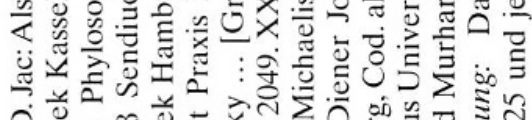

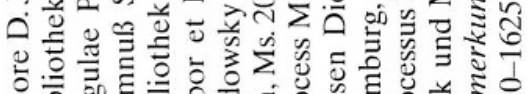

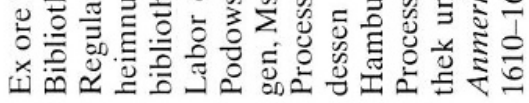

$\because$ 으 
17. Jahrhunderts in Deutschland und Frankreich wirkte ${ }^{24}$. Die Handschrift KI muss im Zeitraum 1604-1618 entstanden sein, denn sie verarbeitet einerseits Gedankengut des Sendivogius-Traktats «De Lapide Philosophorum», andererseits finden sich ihre lateinischen Passagen wörtlich übersetzt in HI.

Der Verfasser von K I war sichtlich bemüht, die spekulative SendivogiusRezeptur praktisch-chemisch umzusetzen, allerdings ohne grossen Erfolg. Für die Bereitung des Lapis Philosophorum verlangt er sogar 17monatiges Erhitzen in einem Glasofen [sic]. Immerhin werden hier der «mercurius Philosophorum» und das «aqua salis nitri», gewonnen durch trockene Destillation von «terra virginea», einheitlich als Königswasser gedeutet. Es soll zwar nur das «lebendige Gold» auflösen und nicht das gemeine, aber wir lesen dazu die kritische Anmerkung: «Ego contrar[iu]m vidi ap[ud] Comitem Wilhelmum à Solms» ${ }^{25}$. Aus der Tatsache, dass der Verfasser von K I Zugang zum Hofe des Grafen von Solms ${ }^{26}$ hatte, kann gefolgert werden, dass das Manuskript im hessischen Raum verfasst wurde.

Fragmente bzw. Modifikationen der Processus-Urfassung finden sich in einigen zeitgenössischen Traktaten ${ }^{27}$ und in einem Brief, den ein sonst nicht bekannter Christian von Wildeck am 10. Oktober 1623 aus Köln an seinen Schwiegervater gerichtet haben soll (gedruckt 1699) ${ }^{28}$.

2. Manuskripte, deren Herkunft Jan Bodowsky zugeschrieben wird, dem Kammerdiener oder Haushofmeister von Sendivogius, der ihm als Gehilfe bei alchimischen Experimenten und Demonstrationen diente: E und H II, letzteres mitgeteilt von Joh. Podasci (wohl verderbt aus Bodowsky). E nennt als weitere Bezugsperson noch den Theosophen und Alchimisten Grossche-

24 Nach einer freundlichst gewährten Information von Herrn J. Paulus/Heidelberg war Jacob Alstein als Rat des französischen Königs Heinrich IV. tätig und mit vielen europäischen Alchimisten bekannt, darunter mit Joseph Duchesne. Ob er allerdings an der Erstausgabe des Sendivogius-Traktats «De Lapide Philosophorum» mitwirkte, wie F. Secret (Ambix 26 [1979] 2, S. 83) behauptet, ist fraglich. Im Impressum und Vorwort der o. O. (Prag?) erschienenen Erstausgabe 1604 ist der Name Alstein nicht erwähnt.

$25 \mathrm{~K} \mathrm{I}, \mathrm{Bl} .23 \mathrm{v}$.

26 Bei dem erwähnten Grafen von Solms könnte es sich um Wilhelm I., Graf zu Solms-Greifenstein (1570-1635, regierte ab 1602) handeln, über dessen alchimische Interessen allerdings nichts bekannt ist. Vgl. Rudolph Graf zu Solms-Laubach: Geschichte des Grafen- und Fürstenhauses Solms. Frankfurt/M. 1865, S. 66-70 u. Tabelle IV.

27 Vgl.z. B. Ioann. Staricius: Auslegung des Manuals Philippi Theophrasti Paracelsi. Item/Theorie und Praxis der Hermophrodito flore Sappharico Magni Georgij Phaedronis ..., Magdeburg 1624, hier unter dem Titel «Das wahre einige Universal / von Saltzburg überkommen 1619».

28 Die phantasievolle Prozessbeschreibung, die ganz ohne Edelmetalleinsatz auskommt und sich auf die Gewinnung eines Universallösungsmittels beschränkt, verrät wenig alchimische Sachkenntnis. Sie wurde abgedruckt von J. H. Cohausen: Tentaminum Physico-Medicorum Curiosa Decas de Vita Humana theoretice et practice per Pharmaciam prolonganda, Osnabrugi 1714 ( ${ }^{1} 1699$ Cosfeldiae), S. 94-96. 
del $^{29}$. Es müsste, nach den Lebensdaten Grosschedels zu urteilen, bis spätestens 1635 enstanden sein. Weitgehend gleichlautend mit E, allerdings ohne die theoretische Einleitung und ohne Bezugnahme auf Bodowsky, ist die Handschrift G I. Bei ihr könnte es sich um eine Abschrift von E handeln.

H II nimmt insofern eine Sonderstellung ein, als der Text aus sehr knappen lateinischen Laborvorschriften besteht, mit einer Vielzahl schwer entzifferbarer Abkürzungen und alchimischer Fachtermini. Ein Zusammenhang mit E ergibt sich hier aus übereinstimmenden Schreibervermerken, nach denen anzunehmen ist, dass der/die Schreiber im nordwestlichen Böhmen ortskundig war(en).

Alle genannten Manuskripte entsprechen im Ablauf der einzelnen Verfahrensschritte des Grossen Werkes bereits Drfg. Becher I.

3. Manuskripte, die, mit ausführlichen theoretischen Begründungen und praktischen Kommentaren versehen, als direkte Vorlagen für die Becherschen Druckfassungen in Betracht kommen: K II und Kr für Drfg. Becher I, G II für Drfg. Becher II. Es handelt sich um durchgehend deutschsprachige Texte mit gelegentlichen lateinischen Passagen und Fachausdrücken. Sie entstanden wahrscheinlich in der 1. Hälfte des 17. Jahrhunderts (gesichert für K $\mathrm{II}^{20}$ ), jedenfalls aber lange vor Erscheinen der Becherschen Druckausgaben).

Es erhebt sich nun die Frage nach dem wirklichen Verfasser des «Processus D. Michaeli Sendivogii super centrum universi, seu Sal centrale», wie er uns in den Handschriften-Texten und den Becherschen Druckfassungen vorliegt.

Für die beiden Processus-Urfassungen steht die geistige Urheberschaft des polnischen Alchimisten ausser Frage. Die Mitwirkung seines Dieners Jan Bodowsky an den verbesserten Prozeduren von E und $\mathrm{H}$ II ist rein spekulativ $^{30}$; sie wird hier nicht weiter diskutiert. Wir haben Anlass zu der Vermutung, dass die Processus-Urfassung noch vor 1632 im bayerisch-hessischen Raum, wahrscheinlich am Hof des hessischen Landgrafen Moritz von Kas$\mathrm{sel}^{31}$, zu jener eigenartigen Lapis-Rezeptur umgestaltet wurde, die mit Sendivogius' spekulativer Originalprozedur nur noch sehr wenig gemein hat.

29 In Grosschedels gedruckten spekulativ-alchimischen Traktaten «Trifolium Hermeticum, oder Hermetisches Kleeblatt I-III» und «Proteus mercurialis geminus, exhibens naturam metallorum ...» (beide Frankfurt/M. 1629) wird der «Processus universalis» nach Sendivogius nicht erwähnt.

30 P. Borel: Trésor de recherches et antiquités Gauloises et Françoises en ordre alphabet, Paris 1655, S. 474-478, druckte eine Sendivogius-Vita, die angeblich von Budowski/Bodowsky stammt. Danach verstarb dieser kurz nach Sendivogius' Tod, also 1636 oder 1637, auf einer Reise in Preussen. R. Bugaj ([11a], S. 185) hat diese Angabe übernommen, räumt aber ein, dass Borel ein unzuverlässiger Informant ist. Zur Fragwürdigkeit der Borelschen Aussagen vgl. man auch D. G. Morhof: De metallorum transmutatione ..., Hamburg 1673, S. 150.

$31 \mathrm{Vgl}$. B. T. Moran: The alchemical world of the German court. Occult philosophy and chemical medicine in the circle of Moritz of Hessen (1572-1632). Sudhoffs Arch., Beiheft 29 (1991). 
Ein persönlicher Einfluss von Michael Sendivogius auf die Prozessvorschriften der Gruppe II/III-Manuskripte ist stilistisch und inhaltlich nicht nachweisbar. Ebenso gibt es keine Beweise für die praktische Mitwirkung von Sendivogius in hessischen Alchimie-Laboratorien ${ }^{32}$. Daher hat wohl auch W. Hubicki, der Altmeister der polnischen Alchimieforschung, den «Processus ... super ... Sal centrale» nicht unter den Sendivogianischen Schriften aufgeführt ${ }^{33}$.

\section{Johann Joachim Becher als Manuskript-Herausgeber}

Welches Manuskript diente Johann Joachim Becher nun als Vorlage für seinen «Processus D. Michaelis Sendivogii super centrum universi, Seu Sal centrale»?

Becher macht im Vorwort zum «Chymischen Glücks-Hafen ...» nur allgemeine Angaben zu Herkunft und Alter seiner Druckvorlagen ${ }^{34}$. Er merkte an, dass er sie seit mehr als 20 Jahren (also etwa seit 1660) gesammelt und unbearbeitet zum Druck gebracht habe, ferner, dass es sich um Aufzeichnungen von Laborexperimenten handele. Für die Herkunft nannte er ausser dem Laboratorium am Prager Hof Kaiser Rudolfs II. vorwiegend oberdeutsche Alchimistenlaboratorien.

Wie ein genauer Textvergleich zeigt, muss Drfg. Becher I aus K II oder Kr hervorgegangen sein; eine Unterscheidung zwischen beiden Handschriften ist nicht möglich. Entgegen seiner Ankündigung dürfte Becher die Druckvorlagen allerdings nachhaltig bearbeitet haben. So finden wir zusätzliche Hinweise auf die Alchimie des Sendivogius eingefügt, Versuchsanleitungen gestrafft und lange deutschsprachige Passagen frei ins Lateinische übersetzt. Sinnentstellende Übertragungsfehler blieben dabei nicht aus ${ }^{35}$.

32 Der Besuch von Sendivogius im chymiatrischen Laboratorium von Prof. Hartmann in Marburg 1616, wie von R. Bugaj ([11a], S. 140) angenommen, ist durch Quellen nicht bezeugt, ebenso nicht sein Aufenthalt am Hofe des Landgrafen Moritz von Hessen-Kassel. Vgl. dazu W. Hubicki, in: Studia i Materialy z Dziejów Polskiej 12 (1968), S. 79-103, hier S. 98.

33 Vgl. W. Hubicki, in: Proc. 10th Int. Congr. Hist. Sci., Ithaca/Philadelphia 1962, Vol. II (publ. 1965), S. 829-833; Ders., in: Actes XI Congr. Int. Hist. Sci., Warszawa/Toruń/ Kraków 1965, T. 4 (publ. 1968), S. 31-35.

34 Weder in der umfassenden Becher-Biographie von H. Hassinger (Johann Joachim Becher, 1635-1682. Ein Beitrag zur Geschichte des Merkantilismus. Wien 1951), noch in Bechers nachgelassenen Schriften (Univ.-Bibliothek Rostock, Varia Becheriana, Mss. var. 1.1-3, 2) oder in seinen gedruckten Altersschriften konnte ich Angaben zu seiner Sammel- und Publikationstätigkeit finden.

35 In Drfg. Becher I, S. 231, fallen ins Auge die missverstandenen Passagen «... nach der Geburt gereiniget ...» und - besonders irreführend - «... der himmlische und sichtbare Gott der Natur ...» Die handschriftlichen Vorlagen haben richtig «... nach gebuer gereiniget ...» und «... der Himlische Unsichtbare Geist der Natur ...» (Kr, Bl. 77r). 
Einige Textbeispiele mögen die Beziehung zwischen Original und Druckfassung verdeutlichen:

\section{Manuskript K II, Bl. 164r, v \\ Athanor}

«Es seindt in dem Athanor 3 Kugeln, die Erste die gröste, gantz: die andre oben auf gelochert wie die 〈Feuer> pfannen, da man die bette mit warmet, damit der dampf des $<$ Wasser> $\beta$ hindurch tringen müge, vnd die dritte ist die holtzerne Eichene Kugel. In der Inneren Kugel geschicht die putrefaction, im vaporischen 〈Feuer», Vnd muß in der ersten Kugel alzeit <Wasser $>$ sein, weil die putrefaction wehret ...»

\section{Manuskript Kr, Bl. 79r}

Folgett die ander Composition

«Das Rote Körnlein, welches der Philosophorum lebendiges 〈Gold〉 ist, wäge genaw ab, thu Zehen mahl so viell oder Schwer menstrui Dazu, thue alles in ein kleine phiol, des $2 / 3$ lehr stehe, sigilla \& procede in Athanore, ut sequitur.»

\section{Drfg. Becher I, S. 234 \\ Usus Athanoris}

«Im Athanor seynd 3. Kugeln /die erste ist die gröste / und gantz. Die andere oben auff gelöchert / daß der Dampff vom warmen Wasser hindurch tringen möge. Die dritte ist die hölzerne eichene Kugel / in der inneren Kugel beschicht die putrefaction im vaporischen 〈Feuer> / und muß in der innersten Kugel allzeit Wasser seyn / so lang die putrefaction durirt und wäret ...»

\section{Drfg. Becher I, S. 235}

Compositio magni operis

«Rec. hoc granum rubicundum, quod est ‘Aurum〉 Philosophorum vivum, pondera valde attente $\&$ diligenter, Appone partes decem ponderi de menstruo Philosophico superius facto, impone phiolae, quae tres partes habeat vacuas, \& unam partem solum à materia occupatam [sic]. Sigilla hermetice, pone in Athanor, \& procede, ut sequitur.»

Eine ähnliche Beziehung existiert auch zwischen Manuskript G II und Drfg. Becher II. Für die Druckwiedergabe wurde der handschriftliche Text, der ausführliche Kommentare enthält, auf ca. ein Drittel seines Umfangs zusammengestrichen.

Auch hierfür ein Textbeispiel:

\section{Manuskript G II, BI. 2r}

«Dieses unser Sal naturae oder terrae, ob es wohl dem Ansehen nach nur eines zu seyn scheinet, so hat es doch 3 unterschiedliche Salia in sich, denn vor sich selbsten ist es erstlich unser philosophisches Saltz Nitri naturae et terrae, darinnen der Spiritus mundi häuffig stecket, nicht gar flüßig und nicht gar fix, sondern hat eine Mittel-Natur ...»

\section{Drfg. Becher II, S. 236}

Modo intrabimus laboratorium chymicum

«Dieses unser «Sal〉 nitri, terrae virgineae, ob es wol dem Ansehen nach nur ein Ding zu seyn scheinet / hat es doch 3. unterschiedliche salia in sich / dann vor sich selbsten ist es ein köstlich und Philosophisches «Nitrum〉/ darinnen der Spiritus mundi steckt / nicht gar fix / sondern mittelmässiger Natur.»

Abschliessend sei noch einmal auf die Chemie des «Processus universalis» nach Sendivogius eingegangen.

Wie schon oben erwähnt, muss im Reaktionsgemisch der Lapis-Präparation stets ein ausreichender Anteil Silber anwesend sein (für Ag$\left[\mathrm{AuCl}_{4}\right]$ theo- 
retisch $\mathrm{Ag} / \mathrm{Au} \approx 1: 1,9$ ), damit sich die edelmetallreiche $\mathrm{Ag} / \mathrm{Au}-$ Komplexverbindung bilden kann. Tatsächlich fordern sowohl die Originalrezeptur von Sendivogius wie die beiden Processus-Urfassungen den Einsatz von Silber und Gold im Mengenverhältnis 2:1, also Ag in starkem Überschuss. Die meisten der weiterentwickelten Prozessvorschriften sehen immerhin noch ungereinigtes, silberhaltiges Naturgold ${ }^{7}$ vor: «gewachsen Gold» (E, G I) oder «klein geschlagen natürlich 〈Gold»» (K II, Kr).

Becher hat seine Druckvorlagen entschieden verschlimmbessert, denn er verlangt in Drfg. Becher $\mathrm{I}^{36}$ «<aurum» corpore, purgatum naturale». Übertroffen wird er hierin noch vom G II-Verfasser, der statt Naturgold «... fein Gold, so auf das allerhöchste fixiret, und 6 oder 7 mahl durch das Antimonium gegossen» verwendet ${ }^{37}$. Diese Reagenzien können natürlich keinen kristallinen Lapis Philosophorum liefern.

Da optimale Reaktionsbedingungen (wozu auch die richtige Erhitzungsdauer und -temperatur gehören) historisch wohl nur in den wenigsten Fällen verwirklicht wurden, darf nicht verwundern, wenn es in einem der handschriftlichen Texte zum «Processus universalis» resignierend heisst ${ }^{38}$ :

«Marcus Friederich Rosen-Creuzer ${ }^{39}$ hat diesen Process mit einem von Adel, laboriert, nichts ausgericht, verwirfft ihn ganz und gar.»

36 Drfg. Becher I, S. 234.

37 G II, Bl. 5r. - Schmelzen mit «Antimonium» (Spiessglanz, $\mathrm{Sb}_{2} \mathrm{~S}_{3}$ ) war eine in der frühneuzeitlichen Probierkunde gängige Methode zur Goldraffination.

38 G I, S. 55 (Randnotiz).

39 Marcus (Marx) Friederich Rosencreutzer, gestorben nach 1640, war das Pseudonym des Nürnberger Mathematikers und Theologen Franz Ritter, unter dem er jährlich einen «Wurtzund Kräuter-Calender» herausgab (nach J. G. Doppelmayr: Historische Nachricht von den Nürnbergischen Mathematicis und Künstlern ..., Nürnberg 1730, S. 96/97). 